\title{
Fall detection for robotic endoscope holders in Minimally Invasive Surgery
}

\author{
Jesus Mago ${ }^{1,2}$, François Louveau ${ }^{2}$, Marie-Aude Vitrani ${ }^{1}$
}

\begin{abstract}
Classic Minimally Invasive Surgery (MIS) is an ergonomic burden for assistants and surgeons. The former need to adopt uncomfortable positions for hours while holding a camera to track the latter's gestures inside the patient. This incurs assistant's muscle fatigue which can lead to tremor or drift of the video feedback. A backdrivable robotic holder can be attached to this device in order to compensate its weight. This allows the user to place the camera at a desired position which the robot will steadily keep once he/she releases it. However, endoscopic cameras present difficult-to-model accessories whose gravity parameters can change during the same surgery. If these changes are not foreseen by the gravity model of the robot this results in a fall of the endoscope each time it is released. Therefore, it is desired to firstly detect if there is a fall in order to be able to correct it. In this article a fall detection method for a comanipulated robotic endoscope holder is proposed. It evaluates smoothness of the robot end effector trajectory to identify whether the user manipulates the instrument or it has been released and poorly compensated. An experiment was carried out with 10 subjects where 240 releases of the endoscope were performed while it was poorly compensated. The algorithm succeeded to detect the falls with sensitivity up to $99.17 \%$.
\end{abstract}

\section{INTRODUCTION}

Laparoscopy is a minimally invasive surgery that allows surgeons to operate abdominal and pelvic organs through small incision making use of long rigid instruments. The gestures inside the patient can be monitored thanks to a video feedback obtained from a tubular rigid camera called endoscope or laparoscope [1]. In classic laparoscopy this camera is held by an assistant at a position previously indicated by the surgeon. Several problems are intrinsic with this approach: the positions that assistants have to adopt in order to hold the camera are both uncomfortable and cumbersome for the constrained work-space of the surgery. What is more, holding the camera for hours incurs muscle fatigue which leads to tremor or drift in the video output. To ease this aspect, a robotic arm can be attached to the camera to steadily hold it at any desired position.

\section{A. Laparoscopic surgery and robotic holders}

In literature several robotic solutions have been proposed to address this problem. An extensive review on robotic endoscope holder can be found in [2]. However, these solutions present a common drawback which is the utilization of high-gain control laws to maintain a desired position. This represents a safety risk as the robotic arm can apply harmful forces over tissues in case of unexpected movements of the

\footnotetext{
${ }^{1}$ Sorbonne Université, INSERM U1150, CNRS UMR 7222, Institut des Systèmes Intelligents et de Robotique (ISIR) F-75005, Paris, France,

${ }^{2}$ Haption SARL
}
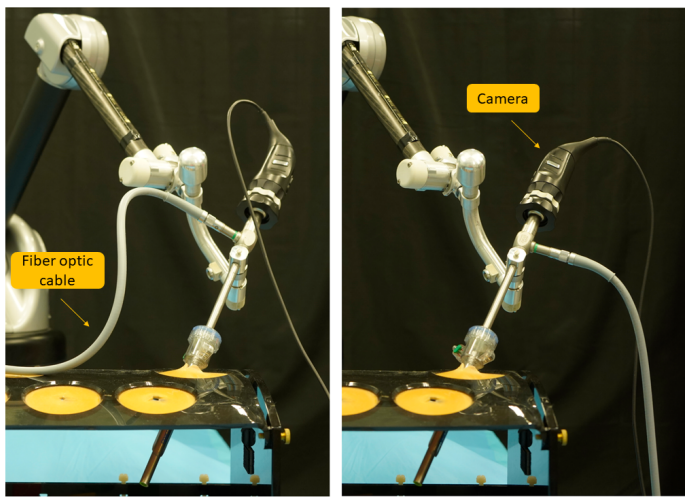

Fig. 1: Different configurations of fiber optic cable.

patient. In addition, these are solutions based on robotic arms with low or none backdrivability which constraints their usage to only teleoperation approaches (voice commands, joysticks, pedals, buttons etc.).

\section{B. Problem statement}

We presented in [3] a fully backdrivable robotic endoscope holder that features two modes: free and locked mode. In free mode the user can place the camera by hand at any desired position in a comanipulation fashion [4]. After the camera is released, the robot steadily keeps the release position thanks to a model-based gravity compensation. Once the device is kept immobile for more than a pre-set time $\Delta t$ (Usually set around $3 \mathrm{~s}$ to $10 \mathrm{~s}$ ), the control law switches to a locked mode to maintain this last position by means of a compliant PID.

The problem with this approach arises when the gravity model does not correspond accurately to reality. In this case the user can not release the endoscope before it is locked as, otherwise, it will fall from the release position. This situation imposes an inconvenient trade-off: setting $\Delta t$ too long, represents a disruption for the normal handling of the endoscope, i.e., the user has to spend an idle time whenever she/he wants to release the camera. On the other hand, if this time is set too short, there is the risk of false positives each time the user reduces his/her handling velocity, locking the robot when it is not desired.

Approaches that involve pedals or buttons that switch between locked mode and free mode can be solutions as well. However these kinds of approaches would require, firstly an additional hardware that could be either cumbersome or incompatible with the usual operating room's equipment. Secondly, these would require an additional cognitive requirement from the user to synchronise the activation of 
the button or pedal with his/her actual manipulation. On the other hand, a global viscous field can be set to counteract the fall of the endoscope. Nevertheless, such a force would attempt against the transparency of the robot while being comanipulated.

Therefore, an accurate gravity model of the endoscope is indispensable. However, developing mathematical models of these devices is a challenging task: they usually feature difficult-to-model elements as, for instance, the fiber optic cable attached to them. This is a deformable element that weights around $250 \mathrm{~g}$. Depending on the surgery and the space available, this cable can be placed in several locations, hanging completely or partially from the camera. Take for instance the situation shown in Fig.1. In the left picture, center of mass of the cable is at the left of the camera axis and its weight is shared between camera and a surface, whereas for the right image the weight is supported mainly by the camera and the cable's center of mass is at the right. Each of these configurations represent a different gravity model. On this account, it is of interest to have a solution that could adapt to this kind of uncertainties in real time in order to avoid any fall after the release of the camera.

\section{Adaptive compensation methods}

In literature several algorithms to address uncertainties in gravity models have been proposed. They can be divided in two categories: mechanical and software-based solutions. Mechanical solutions consist in using springs or counterweights to guarantee a constant potential energy of the system. An adaptive behavior can be featured adding actuators to these mechanisms. They can be used to change the lever arm of counterweights or to change the precharge load of springs. A review on these solutions can be found in [5]. The main drawback of these approaches is the need of cumbersome and heavy elements in order to compensate for changes in the payload.

On the other hand, software-based solutions are of interest as they are lightweight. Methods as [6], [7], [8] include approaches based on fuzzy control and sliding modes algorithms for dealing with high variability of payload while guarantying stability. The main limitation with these methods is the need of computing a set-point or trajectory in order to compensate for anomalies in the gravity model. These solutions are not adequate for the free mode previously explained as it is not possible to define a set-point or trajectory when the idea is to allow the user to freely displace the end-effector of the robot.

There are other approaches that do not require the constant computing of trajectories or set-point. In [9] an Extreme Machine Learning (EML) is used to develop an adaptive controller for a lightweight robot. It requires a first phase where data from random configurations of the robot with a known payload is collected to estimate an inverse equilibrium model. In [10] a method is developed to automatically estimate apparent mass of a 3D haptic device. In a off-line phase they estimate a 3D mapping of apparent masses for the workspace of the robot for each new unknown payload. The main drawback with these kind of solutions is the necessity of training sessions for each new payload. These solutions are not suitable for compensating for an endoscope since it is not convenient to go through a training set of positions each time the gravity configuration of the system change, e.g when moving the optic fiber cable.

In this article, an online method to detect a fall due to a wrong gravity compensation is proposed. It is implemented in a cable driven robot used as endoscope holder. It consists in an algorithm that calculate smoothness of the robot endeffector trajectory to detect whether there is a fall after the user releases the camera. In last section possible approaches are commented that can be implemented once a wrong gravity compensation is detected to avoid the fall or update the gravity model.

The rest of the paper is organized as follows: Section II presents the device and the methodology adopted to record data of manipulation and falls of the endoscope. First, the general set-up is presented, then the Achilles robot is described and finally the experimental protocol to acquire data is detailed. Afterwards, The fall detection algorithm is presented in Section III. First, the chosen criterion is presented, followed by information on the algorithm implemented in practice, including the method of setting its parameters. Results of the proposed method are presented and discussed in Section IV. In Section IV-D two approaches for compensate the falls are introduced as perspectives. Finally, conclusions are presented in Section V.

\section{MAterials AND METHODS}

The following experiment aims to acquire data during realistic endoscope manipulation and release. The aim is to verify that the proposed criterion, proposed in Section III, is capable of distinguishing between movements of the endoscope when the user manipulates it or when it presents a free fall.

\section{A. Overall Setup}

The overall setup used, described hereafter, is the "Surgical Cockpit platfor" [11] that allows to reproduce a realistic situation of robot-assisted minimally invasive surgery. It consists in an operating room mockup, see Fig.2.

The operating room was composed by the following elements:

- Laparotrainer: a practical surgical model whereby surgeon can practice laparoscopic techniques.

- Targests: 6 different targets were placed inside the laparotrainer as shown in Fig. 2 bottom right corner.

- Endoscope: It is used to visualise the objects inside the laparotrainer. It is used with an optic fiber cable attached to it.

- Large Endoscopic screen: this screen show the video capture from the endoscope

- Achilles: the endoscope-holder presented in sec.II-B. 


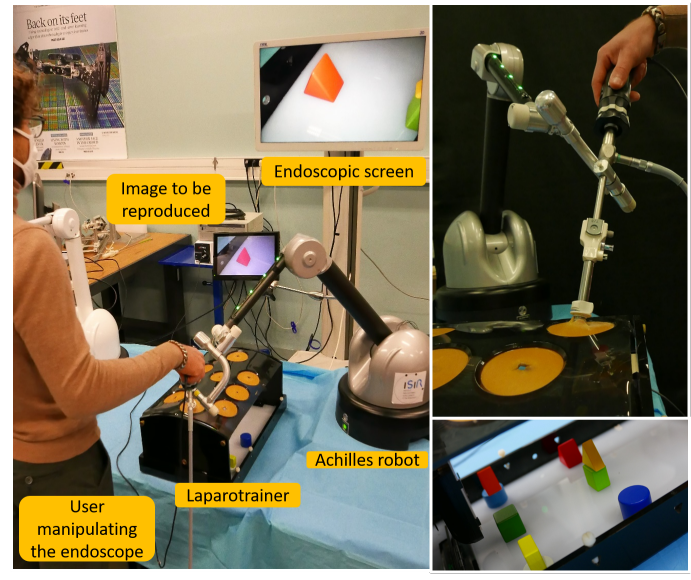

Fig. 2: Overall set-up. On the left is the general view, on the top right a focus on the endoscope, on the bottom right the inside view of the laparotrainer with the objects to visualize

\section{B. Achilles: kinematics and actuation}

The robotic manipulator Achilles manufactured by HAPTION and initially designed to serve as a high-force haptic interface, is employed as a comanipulated laparoscopeholder, see Fig. 2 top right corner.

Fig.3 shows the kinematics of the device, made of 6 pivot joints:

- the last three joints, whose axes intersect at a point $W$, form a wrist.

- the first three joints are used to position the point $W$.

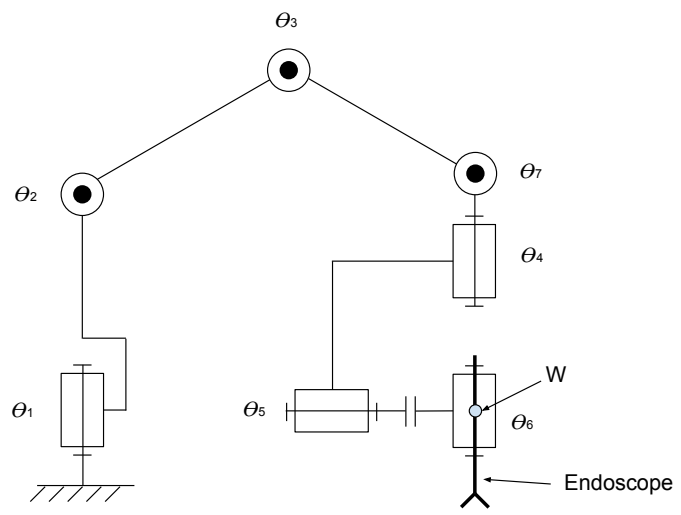

Fig. 3: Achilles kinematics

The geometry is fully defined thanks to the DH parameters grouped in Table I

Joints 1, 2, 3 are active (motorized) while joints 4, 5, 6 are passive (free rotations). Joint 7 is a discrete joint that allows fixed positions in an angular range of motion of $120^{\circ}$.

The end-effector is equipped with a magnetic interface that allows to attach the endoscope, see Fig. 2 top right corner.

This robot is designed to comanipulate the endoscope. For this purpose, the robot has a "free mode" control law, based on a model-based gravity compensation that allows the operator to move the endoscope without constraints (details
TABLE I: DH parameters of the device

\begin{tabular}{||c|c|c|c|c||}
\hline Joint & $\alpha_{i-1}$ & $a_{i-1}$ & $d_{i}$ & $\theta_{i}$ \\
\hline 1 & 0 & 0 & 0 & $q_{1}$ \\
2 & $\pi / 2$ & 0 & 0 & $q_{2}$ \\
3 & 0 & $l_{1}$ & $-l_{3}$ & $q_{3}$ \\
4 & 0 & $l_{2}$ & $l_{3}$ & $q_{4}$ \\
5 & $\pi / 2$ & 0 & $l_{4}$ & $q_{5}$ \\
6 & $\pi / 2$ & 0 & 0 & $q_{6}$ \\
\hline
\end{tabular}

are given in appendix, see also [3] and [11]). Notice that, for the experiment presented in this paper, the gravity model of the robot was set to compensate the weight of the endoscope alone, i.e. the weight of the optic fiber cable attached to it is not being compensated. As a result, when the user releases the endoscope, it falls instead of remaining at the release position.

A viscosity field was implemented in order to dampen down the velocity of the end-effector when this is released [11]. It consists in a Cartesian force mapped at the robot end-effector defined as shown in (1):

$$
\mathbf{F}_{v}=b * \mathbf{v}
$$

where $b$ is a parameter that varies in function of the norm of the end effector velocity:

$$
b= \begin{cases}b_{\max } & \text { if }\|\mathbf{v}\| \leq v_{\min } \\ b_{\min } & \text { if }\|\mathbf{v}\| \geq v_{\max } \\ b_{\max }+\frac{\|\mathbf{v}\|-v_{\min }}{v_{\max }-v_{\min }} & \text { otherwise }\end{cases}
$$

Notice that function $b$ requires four parameters to be tuned, $b_{\max }, b_{\min }, v_{\min }$ and $v_{\max }$.

This viscous field is intended to be active only when the release is imminent. Surgeons and assistants usually release the endoscope once they get a stabilized video, i.e, when velocity is near zero. Therefore, the values $v_{\min }$ and $v_{\max }$ were set to $0.01 \mathrm{~m} \mathrm{~s}^{-1}$ and $0.012 \mathrm{~m} \mathrm{~s}^{-1}$ respectively in order to set the viscous field at its maximum value just before the release, damping down the fall. On the other hand, when user makes movements above $v_{\max }$, the viscous forces disappear, i.e $b_{\min }=0$, avoiding any perturbation in the user gesture.

\section{Data acquisition Protocol}

Ten naive subjects were asked to perform the experiment which is explained in the following:

A reference image is shown to the user. It shows one of the target with a specific depth and orientation of the camera. A small screen (see Fig.2) is added to the operating room mock-up to provide the user with this image. The subject is asked to reproduce the reference image in the large screen (Endoscopic screen in Fig. 2). As the robot is set in free mode, the subject can freely place the endoscope in order to get the correct depth and orientation of the image. Once he/she thinks the right configuration is attained, the subject has to release the endoscope. The subject is asked to inform the moment just before he/she was about to release the endoscope. Each subject is asked to reproduce 12 different images (see Fig.4). 
In order to asses how much influence the parameter of viscosity has on both rate of detection and perturbation on user handling, the experiment is repeated for two values of $b_{\text {max }}: 50 \mathrm{Ns} \mathrm{m}^{-1}$ and $100 \mathrm{Ns} \mathrm{m}^{-1}$.

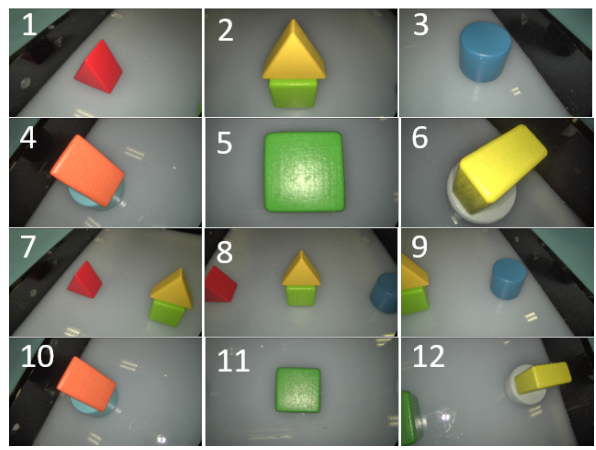

Fig. 4: Images to be reproduced by the user manipulating the endoscope

a) Ground truth: in order to have a ground truth the subject is asked to inform the moment just before he/she was about to release the camera. At that moment a label was set to identify the moment before and after the release. The exact moment where the release happened is then manually located, in a off-line phase, by inspection of the end-effector's Cartesian velocity. It should be noted that during these tests, it is observed that, depending on the configuration, when the user lets go of the endoscope, it falls more or less quickly and stabilizes more or less far from the initial position.

b) Data recorded: A total of 240 falls are observed (12 targets, 10 subjects, 2 viscosity conditions). For each subject, during each experiment, the data recorded includes:

- an array of the end-effector's Cartesian velocity $\mathbf{v} \in$ $\mathbb{R}^{3 \times s}$

- the ground truth array $L \in \mathbb{R}^{1 \times s}$. $L$ is a boolean array containing 1 for samples were it was manually labeled a fall and 0 otherwise.

Notice that the robot Achilles has a sampling frequency of $1 \mathrm{kHz}$ which means that a new sample is recorded at each millisecond. The value $s$ represents the duration in milliseconds of the whole experiment including 12 falls.

\section{FALL DETECTION ALGORITHM}

In this paper the reasoning of the proposed method is based on the hypothesis that the movement of a falling object attached to the robot can be differentiated from those originated by a human manipulating the robot in free-mode. In other words, we count on the fact that trajectories due to wrong compensations are predominated by low frequencies - as they are exited only by low-frequency forces, e.g. the non-compensated weight and dynamic friction at the trocar whereas human manipulation generates more saccadic movements and therefore, less smooth. In next section we present a smoothness parameter that can be used to differentiate between these two kind of movements.

\section{A. Assessing smoothness}

In literature, smoothness metrics are used to asses the level of complexity of a curve [12]. For instance, curvature is used as a measure of how sharply a curve bends [13]. Another example is the Spectral Arc Length [12]. It is the opposite of the length along the spectral curve of a movement; it gives an image of the complexity of the movement. In this paper, a new parameter is proposed as possible candidate of smoothness indicator.

Proposed method: Fig.5 represents a trajectory of the end-effector of the robot with respect to the robot base frame $R$.

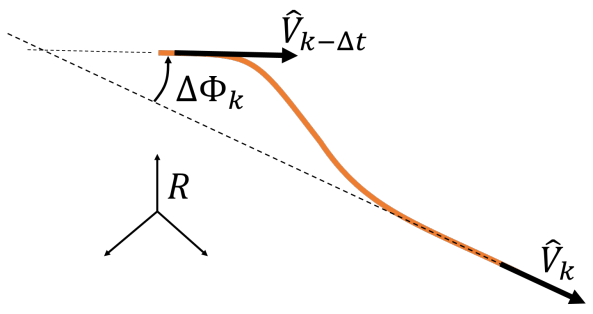

Fig. 5: Illustration of robot's end-effector trajectory

$\hat{V}_{k}$ is a vector parallel to the end-effector velocity at $t=k$ with magnitude equal to one, whereas $\hat{V}_{k-\Delta t}$ is a vector parallel to the end-effector velocity at time $k-\Delta t$ with magnitude equal to one. Let's define $\Delta \Phi(k)$ as the angle between vectors $\hat{V}_{k}$ and $\hat{V}_{k-\Delta t}$ calculated as shown in (3).

$$
\Delta \Phi(k)=\arccos \left(\hat{V}_{k} \cdot \hat{V}_{k-\Delta t}\right)
$$

This function represents the change of direction in velocity during a determined time step $\Delta t$. The lower this value, the straighter the trajectory.

A density criteria was adopted to identify whether the trajectory is smooth or not: If the function $\Delta \Phi(k)$ is below a threshold $\Delta \Phi_{0}$ during $N_{0}$ consecutive time steps the algorithm will declare smoothness of the trajectory and therefore, a fall will be declared. In other words, two functions were defined as shown in (4) and (5):

$$
\alpha(k)= \begin{cases}1 & \text { if } \Delta \Phi(k) \leq \Delta \Phi_{0} \\ 0 & \text { if } \Delta \Phi(k)>\Delta \Phi_{0}\end{cases}
$$

Function $\hat{L}(k)$ is one when a fall is declared and zero otherwise:

$$
\hat{L}(k)= \begin{cases}1 & \text { if } \sum_{i=k-N_{0}}^{k} \alpha(i) \geq N_{0} \\ 0 & \text { if } \sum_{i=k-N_{0}}^{k} \alpha(i)<N_{0}\end{cases}
$$

\section{B. Implemented Algorithm}

The proposed algorithm computes the following 3 steps at each time step of the control loop of the robot: (i) Compute $\Delta \Phi(k)$, (ii) Compute $\alpha(k)$, (iii) Compute $\hat{L}(k)$. 


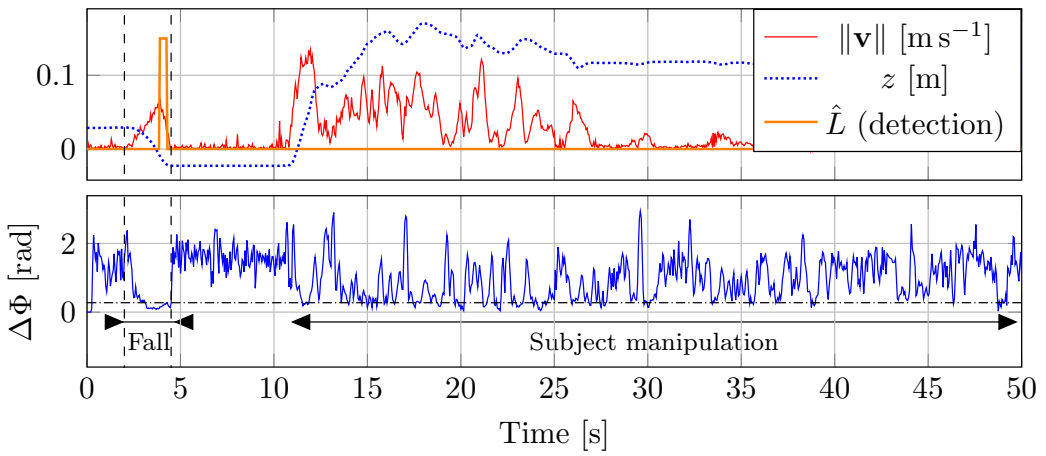

(a)

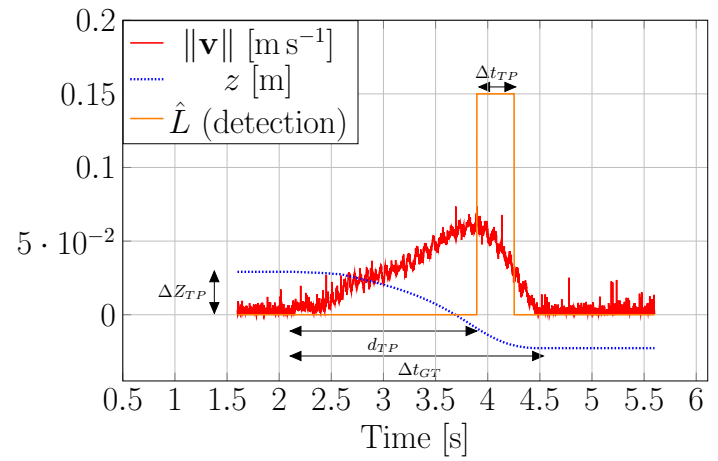

(b)

Fig. 6: (a) Above: Velocity and vertical coordinate of robot end-effector. Below: value of parameter $\Delta \Phi$. The fall is delimited by vertical dashed lines. (b) Behavior of one typical fall. The different performance indicators are shown.

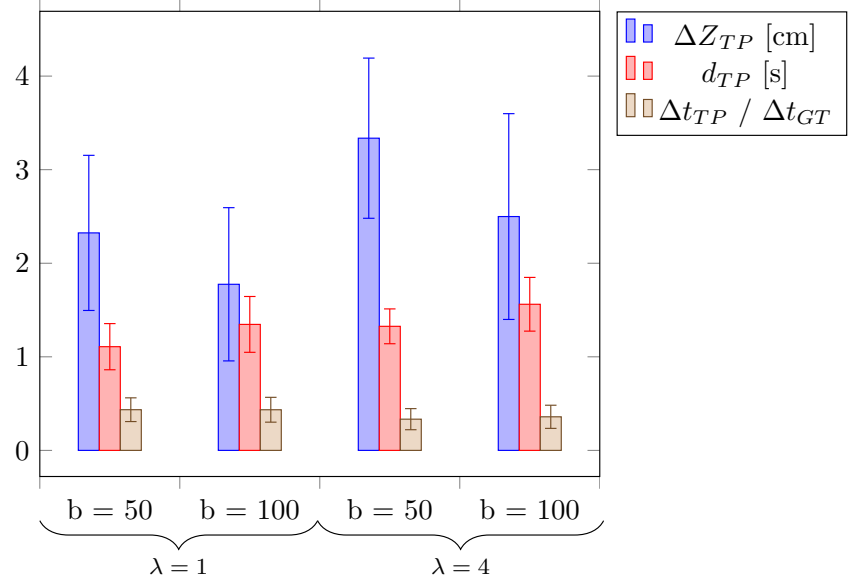

Fig. 7: Average values of performance indicators with respect to parameter tuning and viscosity conditions. Each bar is the mean value and the standard deviation for 120 falls at a given condition of $\lambda$ and $b$.

a) Filtering data: Since human movements have a frequency limit of $20 \mathrm{~Hz}$ [14], the values of end-effector velocities were low-pass filtered with a cutoff frequency at this limit. In this way the method will not be influenced by high frequencies as noise from the encoders. On the other hand, the values of $\Delta \Phi$ presented a noisy behavior which made difficult to compare it with the threshold $\Delta \Phi_{0}$. For this reason a moving average filter of $M$ number of sample points were implemented. Therefore, $M$ is another parameter to set for the implementation of the algorithm.

b) Optimization method to tune parameter: To implement the computation, there are four parameters to tune: $\Delta \Phi_{0}, \Delta t, N_{0}$ and $M$. A data set of $s=344 \times 10^{3} \mathrm{~ms}$ obtained from one of the subjects is used to tune the proposed algorithm. The conditions from which the data was acquired presented viscosity $b=100 \mathrm{Ns} \mathrm{m}^{-1}$. Notice that the data-set from only one subject was used to tune these parameters. This was meant to demonstrate the generalisation of the algorithm over new data-sets.

A prediction function $\bar{L}(k)$ can be defined as follows:

$$
\bar{L}(k)=\left\{\begin{array}{lllll}
1 & \text { if } \quad \sum_{i=k-N_{0}}^{k} \alpha(i) \geq N_{0} & \& & L(k)=1 \\
\lambda & \text { if } \sum_{i=k-N_{0}}^{k} \alpha(i) \geq N_{0} \quad \& \quad & L(k)=0 \\
0 & \text { if } \sum_{i=k-N_{0}}^{k} \alpha(i)<N_{0} \quad \& \quad & L(k)=0
\end{array}\right.
$$

Therefore, an array $\bar{L} \in \mathbb{R}^{1 \times s}$ can be obtained using a set of parameters $p=\left\{\Delta \Phi_{0}, \Delta t, N_{0}, M\right\}$.

An objective function can be defined as follows:

$$
f\left(\Delta \Phi_{0}, \Delta t, N_{0}, M\right)=\|L-\bar{L}\|
$$

Thus an optimization problem is proposed to find the best set of parameters $p$ that minimizes (7), i.e. :

$$
\begin{array}{cl}
\min _{\Delta \Phi_{0}, \Delta t, N_{0}, M} & \|L-\bar{L}\| \\
\text { s.t. } & \Delta t, N_{0}, M \in \mathbb{Z}^{+},
\end{array}
$$

Notice that $\lambda$ in (6) is a weight that can be adjusted in order to increase the euclidean norm shown in (7) each time a false positive is declared. The bigger the value of $\lambda$ the more sensitive is the optimization for false positives.

In order to avoid local minimums the function $f\left(\Delta \Phi_{0}, \Delta t, N_{0}, M\right)$ was mapped using 1000 random draws of parameter sets. Then the set of parameter which gave the minimum value of the function was used as first seed to find the general minimum using the function fminsearch in Matlab.

TABLE II: Parameters obtained for $\lambda=1$ and $\lambda=4$

\begin{tabular}{|l|l|l|}
\cline { 2 - 3 } \multicolumn{1}{c|}{} & $\lambda=1$ & $\lambda=4$ \\
\hline$\Delta \Phi_{0}$ [rad] & 0.1598 & 0.2744 \\
\hline$\Delta t$ [samples] & 150 & 221 \\
\hline$N_{0}$ [samples] & 338 & 787 \\
\hline$M$ [samples] & 325 & 64 \\
\hline
\end{tabular}

Two set of parameters were obtained using two values of $\lambda: 1$ and 4 . These values were considered suitable candidates 


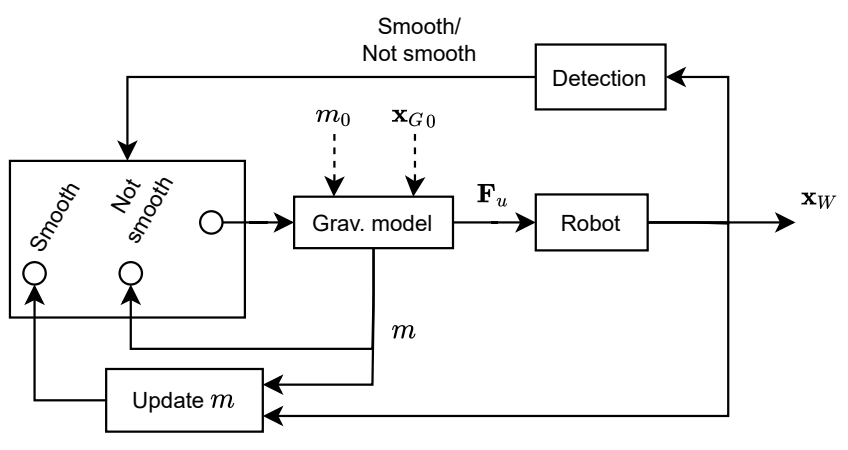

(a)

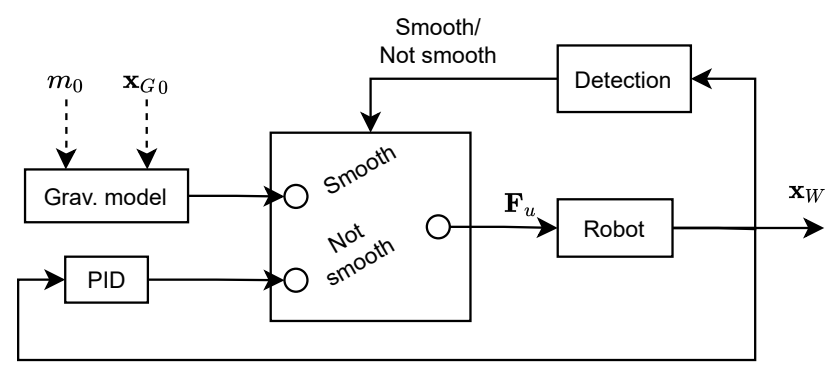

(b)

Fig. 8: (a) Scheme for compensation method to update the gravity model of the robot (b) Scheme for control law to set th

to show the influence of $\lambda$ in the optimization: $\lambda=1$ means that there is no punishment for false positives, therefore, its corresponding set of parameter is expected to lead to results with good sensitivity but lesser specificity. The mirror effect is expected for parameters obtained with $\lambda=4$. Each parameter set are shown in Table II.

\section{EXPERIMENTAL RESUlTS AND DisCUSSIONS}

\section{A. Indicators}

The performance of the algorithm was tested in two experimental condition: viscosity $b=50 \mathrm{Nsm}^{-1}$ and $b=$ $100 \mathrm{~N} \mathrm{sm}^{-1}$. In addition, the influence of the selection of $\lambda$ used in the optimization is evaluated as well. The indicators to evaluate the performance of the proposed method are the following:

- $N_{T P}$ : Number of true positives (TP) during the experiment (the endoscope falls and its fall has been detected).

- $d_{T P}$ : Delay to detect TP (see Fig.6b).

- $\Delta t_{T P}$ : Duration of TP during an actual fall (see Fig.6b).

- $\Delta t_{G T}$ : Actual duration of the falls (see Fig.6b).

- $\Delta Z_{T P}$ : Vertical displacement of the robot end-effector before a fall is detected (see Fig. 6b).

- $N_{F P}$ : Quantity of false positive (FP) during the experiment (a fall is detected, but it should not have been).

- $\Delta t_{F P}$ : Duration of FP.

\section{B. Results}

Fig.6a shows the velocity's end-effector of the robot and its vertical position with respect to the robot base frame (above) and the value of the parameter $\Delta \Phi$ (below) during $50 \mathrm{~s}$ of manipulation by a subject. For this data set the parameters used were the ones obtained using $\lambda=4$ and a viscosity of $b=100 \mathrm{Nsm}^{-1}$. This result can be considered as a typical output where:

1) First, the endoscope is released and presents a fall (delimited by vertical dashed lines)

2) Then, the subject manipulates the endoscope to find the next target (starting from around $\mathrm{t}=10 \mathrm{~s}$ ).

Notice that during the fall, values of $\Delta \Phi$ rapidly decrease below the threshold $\Delta \Phi_{0}$ (depicted with the horizontal dotdashed line). As this condition is steadily kept during more than $N_{0}$ samples, the algorithm declares a fall, i.e. $\hat{L}$ is set to one. On the other hand, $\Delta \Phi$ presents oscillating values and barely reach the threshold $\Delta \Phi_{0}$ when the subject is manipulating the endoscope (starting from around $t=10 \mathrm{~s}$ ).

Table III shows the general performance of the method in terms of sensitivity and specificity for each condition of viscosity and for each different set of parameters. Notice that Sensitivity is calculated as $N_{T P} / P$ and Specificity as $\left(\Delta t_{T N t o t}-\Delta t_{F P t o t}\right) / \Delta t_{T N t o t}$. Where $P$ represents all the actual falls per experiment, i.e. $P=120, \Delta t_{T N t o t}$ represents all the milliseconds where there were not an actual fall and $\Delta t_{F P t o t}$ represents the sum of all the milliseconds where a false positive was declared.

TABLE III: Sensitivity and Specificity for data sets of 120 falls. Number of false positives and their average duration are shown as well

\begin{tabular}{|l|l|l|l|l|}
\cline { 2 - 5 } \multicolumn{1}{c|}{} & \multicolumn{2}{c|}{$\lambda=1$} & \multicolumn{2}{c|}{$\lambda=4$} \\
\cline { 2 - 5 } \multicolumn{1}{c|}{} & $b=50$ & $b=100$ & \multicolumn{1}{c|}{$b=50$} & $b=100$ \\
\hline Sensitivity & 0.9917 & 0.9167 & 0.9750 & 0.9000 \\
\hline Specificity & 0.9976 & 0.9959 & 0.9995 & 0.9991 \\
\hline$N_{F P}$ & 40 & 85 & 9 & 21 \\
\hline$\Delta t_{F P}[\mathrm{~ms}]$ & $167 \pm 176$ & $184 \pm 163$ & $110 \pm 155$ & $150 \pm 140$ \\
\hline
\end{tabular}

The bar chart in Fig.7 shows the performance obtained in terms of parameters $\Delta Z_{T P}, d_{T P}$ and the ratio $\Delta t_{T P} / \Delta t_{G T}$.

\section{Discussion}

Small values of $\Delta Z_{T P}$ and $d_{T P}$ are signs of good performance. This means the method took less time to detect a fall and the end-effector fell less before the detection. The fastest detection time $d_{T P}$ was obtained for the condition $b=50 \mathrm{~N} \mathrm{sm}^{-1}$ and $\lambda=1$. Whereas the smallest fall before detection was presented for condition $b=100 \mathrm{Nsm}^{-1}$ and $\lambda=1$. This is coherent with the selection of $\lambda=1$ : Smaller values of $\lambda$ mean that the optimization method punishes less the false positives. This can be translated in faster reaction time, or in other words, smaller values of $N_{0}$ (see (5)). Following Table III we can conclude that better sensitivity is related with small values of $\lambda$ and viscosity.

On the flip side, small values of $\lambda$ also mean the presence of more false positive: See in Table III that configurations for 
$\lambda=1$ present 40 and 85 false positives, for each value of $b$, whereas configurations obtained with $\lambda=4$ presented only 9 and 21 . However, notice as well that false positives were short in duration, being $184 \pm 163 \mathrm{~ms}$ the biggest average value of $\Delta t_{F P}$.

Low values of sensitivity were observed for high values of viscosity. This can be related to a very specific phenomenon observed during the experiments: for several releases of the endoscope the viscosity was high enough to produce a fall at a particularly low velocity. This movement was characterized by saccadic displacements of the endoscope that can be attributed to a transition phase between the static and dynamic friction within the trocar. When this behavior occurred the method did not identify the fall.

\section{Perspectives}

Once the fall is detected, different strategies can be triggered in order to compensate the fall. Scheme shown in Fig.8a introduce one possible strategy to this aim. When the detection algorithm declares the movement of the robot's end-effector as "smooth", it means that a fall is occurring. While smoothness is declared, the parameter $m$ in the gravity model of the robot - equation (12) - can be updated at each time step until the fall stops. This parameter has to be increased or reduced depending on the robot's end-effector velocity direction when smoothness is declared. This means that, after the first fall, the model will be updated with a value of $m$ that will avoid further falls for future releases of the endoscope. Notice that the same logic can be adopted when the robot overcompensates, i.e., the endoscope presents a rise when it is released.

Another approach is introduced in scheme shown in Fig.8b. Once the detection algorithm declares a fall, the control law of the robot switches to a PID control that will bring the end-effector back to the release position. In order to avoid any harm to the patient, the low-stiffness PID presented in [3] or the PI presented in [15] are good candidates for this purpose. This option can be interesting when, due to small errors in the gravity model of the robot, the endoscope presents small displacements when it is released, meaning that it will not present a risk for the patient. Notice that, in order to implement this scheme, it is necessary to storage the position of the robot's end-effector $\mathbf{x}_{W}$ at each time the endoscope is released. In Fig.8a and Fig.8b $m_{0}$ and $\mathbf{x}_{G 0}$ are seed values for endoscope's gravity parameters of mass and position of center of mass, respectively. $\mathbf{F}_{u}$ represents a Cartesian force applied by the robot at its end-effector by means of (10).

The set-up presented in section II-A presents static conditions, i.e. it does not reproduce physiological movements as the respiration of the patient, which is a low-frequency movement. As a perspective, a set-up that considers this movement should be considered to validate the robustness of the proposed algorithm.

\section{CONCLUSION}

In this article a fall detection for a comanipulated robotic endoscope holder was proposed. An in-vitro set-up and a methodology to record data during the manipulation of the endoscope was implemented. An experiment was carried out with 10 different subjects where 240 releases of the endoscope were performed while it was poorly compensated. Results showed that the algorithm succeed to identify the falls with a sensitivity up to $99.17 \%$. As the proposed approach can be run in real time during manipulation, it can be used into a control law to compensate and stop the fall when it is detected. Finally, two possible strategies to compensate the fall are proposed as perspectives.

\section{APPENDIX : ACHILLES FREE MODE}

The Achilles free mode is based on a control law using the trocar constraint and a gravity compensation algorithm.

1) Trocar constraint: The presence of a fixed fulcrum point $T$ at the trocar imposes two kinematic constraints, reducing the Cartesian DoFs of the system from six to four:

- three to determine the orientation of the laparoscope;

- one for the laparoscope penetration depth.

When the laparoscope is inserted through the trocar, positioning $W$ with the first three joints of the robot allows to control the orientation of the laparoscope axis $\left(W, z_{o p t}\right)$, and its penetration depth $d$. Where:

$$
\begin{aligned}
z_{o p t} & =\frac{1}{\left\|\mathbf{x}_{T}-\mathbf{x}_{W}\right\|}\left(\mathbf{x}_{T}-\mathbf{x}_{W}\right) \\
d & =L-\left\|\mathbf{x}_{T}-\mathbf{x}_{W}\right\|
\end{aligned}
$$

$L$ is the length from $W$ to the distal tip of the laparoscope and $\mathbf{x}_{M}$ stands for the position of a given point $M$. In the practical implementations of the robot controller, $\mathbf{x}_{W}$ is known from the robot kinematic model and the first three joint positions, while $\mathbf{x}_{T}$ is identified on-line thanks to an algorithm described in [16].

Note that because of the passive wrist at point $W$, the first three joint torques $\left(\tau_{1}, \tau_{2}, \tau_{3}\right)$ bijectively map into an equivalent force $\mathbf{F}_{u}$ applied at point $W$, as:

$$
\mathbf{F}_{u}=\mathbf{J}^{-T}\left(\begin{array}{lll}
\tau_{1} & \tau_{2} & \tau_{3}
\end{array}\right)^{T}
$$

where $\mathbf{J}$ is the robot jacobian matrix mapping the three first joint velocities into Point $W$ velocity:

$$
\dot{\mathbf{x}}_{W}=\mathbf{J}\left(\begin{array}{lll}
\dot{q}_{1} & \dot{q}_{2} & \dot{q}_{3}
\end{array}\right)^{T} .
$$

2) Gravity compensation: As explained in the introduction, the robot uses a model-based algorithm, proposed by [11], to compensate for the weight of the endoscope when this has been released. This algorithm consists in mapping a Cartesian force defined as shown in 12 .

$$
\left.\mathbf{F}_{g}=-m\left(\beta\left(\mathbf{I}-\mathbf{z}_{\text {opt }} \mathbf{z}_{o p t}^{T}\right)+\mathbf{z}_{\text {opt }} \mathbf{z}_{o p t}^{T}\right)\right) g, \quad \beta=\frac{\overline{C G}}{\overline{T W}}
$$

Where $\overline{C G}$ is the distance from the tip of the camera to its center of mass and $\overline{T W}$ the distance from the fulcrum point to the wrist $W$. For the experiment presented in Section II, values of $m$ and $\overline{C G}$ were set to $0.487 \mathrm{~kg}$ and $0.360 \mathrm{~m}$ respectively. 


\section{ACKNOWLEDGMENT}

This work was supported by the French state funds managed by the ANR (Agence Nationale de la Recherche) within the Invesissements d'Avenir Program (Labex CAMI) under Reference ANR-11-LABX-0004 and by ANRT (Association Nationale Recherche Technologie).

\section{REFERENCES}

[1] A. J. Senagore, The Gale Encyclopedia of Surgery: A Guide for Patients and Caregivers; 3-volume set. Gale, 2004.

[2] A. Bihlmaier, "Endoscope robots and automated camera guidance," in Learning Dynamic Spatial Relations. Springer, 2016, pp. 23-102.

[3] J. Mago, M. Arico, J. Da Silva, and G. Morel, "Safe teleoperation of a laparoscope holder with dynamic precision but low stiffness." in 2019 International Conference on Robotics and Automation (ICRA). IEEE, 2019, pp. 2693-2699.

[4] G. Morel, J. Szewczyk, and M.-A. Vitrani, "Comanipulation," Medical Robotics, pp. 303-350, 2013.

[5] L. Ciupitu, "Adaptive balancing of robots and mechatronic systems," Robotics, vol. 7, no. 4, p. 68, 2018.

[6] S. Beyhan, Z. Lendek, R. Babuška, M. Wisse, and M. Alcı, "Adaptive fuzzy and sliding-mode control of a robot manipulator with varying payload," in 2011 50th IEEE Conference on Decision and Control and European Control Conference. IEEE, 2011, pp. 8291-8296.

[7] R. Sharma, P. Gaur, and A. Mittal, "Design of two-layered fractional order fuzzy logic controllers applied to robotic manipulator with variable payload," Applied soft computing, vol. 47, pp. 565-576, 2016.

[8] B. K. Yoo and W. C. Ham, "Adaptive control of robot manipulator using fuzzy compensator," IEEE Transactions on fuzzy systems, vol. 8, no. 2, pp. 186-199, 2000.

[9] A. Balayn, J. F. Queißer, M. Wojtynek, and S. Wrede, "Adaptive handling assistance for industrial lightweight robots in simulation," in 2016 IEEE International Conference on Simulation, Modeling, and Programming for Autonomous Robots (SIMPAR). IEEE, 2016, pp. $1-8$.

[10] A. Formaglio, M. Fei, S. Mulatto, M. De Pascale, and D. Prattichizzo, "Autocalibrated gravity compensation for 3dof impedance haptic devices," in International Conference on Human Haptic Sensing and Touch Enabled Computer Applications. Springer, 2008, pp. 43-52.

[11] L. Dong, "Assistance to laparoscopic surgery through comanipulation," Ph.D. dissertation, Paris 6, 2017.

[12] S. Balasubramanian, A. Melendez-Calderon, and E. Burdet, "A robust and sensitive metric for quantifying movement smoothness," IEEE transactions on biomedical engineering, vol. 59, no. 8, pp. 2126-2136, 2011.

[13] M. K. Chmarra, S. Klein, J. C. de Winter, F.-W. Jansen, and J. Dankelman, "Objective classification of residents based on their psychomotor laparoscopic skills," Surgical endoscopy, vol. 24, no. 5, pp. 10311039, 2010.

[14] J. McAuley, J. Rothwell, and C. Marsden, "Frequency peaks of tremor, muscle vibration and electromyographic activity at $10 \mathrm{hz}, 20 \mathrm{hz}$ and 40 hz during human finger muscle contraction may reflect rhythmicities of central neural firing," Experimental brain research, vol. 114, no. 3, pp. 525-541, 1997.

[15] C. Poquet, P. Mozer, M.-A. Vitrani, and G. Morel, "An endorectal ultrasound probe comanipulator with hybrid actuation combining brakes and motors," IEEE/ASME Transactions on Mechatronics, vol. 20, no. 1, pp. 186-196, 2014.

[16] L. Dong and G. Morel, "Robust trocar detection and localization during robot-assisted endoscopic surgery," in 2016 IEEE International Conference on Robotics and Automation (ICRA). IEEE, 2016, pp. 4109-4114. 